\title{
Design and evaluation of the I-SCAN faculty POCUS program
}

Michael Janjigian ${ }^{1 *}$ (D), Anne Dembitzer², Caroline Srisarajivakul-Klein ${ }^{1}$, Khemraj Hardower ${ }^{3}$, Deborah Cooke ${ }^{3}$, Sondra Zabar $^{3}$ and Harald Sauthoff ${ }^{2}$

\begin{abstract}
Background: Point-of-care ultrasound (POCUS) is becoming widely adopted with increasing accessibility of courses. Little is known about the optimal design of the introductory course or longitudinal training programs targeting hospitalists that are critical to success.

Methods: Hospitalists at four academic sites participated in a two-day introductory course and a longitudinal phase comprising clinical POCUS practice, clip uploading with online feedback, hands-on teaching, and monthly ultrasound conferences. Assessments were performed immediately before and after the two-day course and after 1 year.

Results: Knowledge increased from baseline to post two-day course (median score 58 and 85\%, respectively, $p<$ 0.001 ) and decreased slightly at 1 year (median score $81 \%, p=0.012$ ). After the two-day introductory course, the median score for hands-on image acquisition skills, the principal metric of participant success, was 75\%. After 1 year, scores were similar (median score 74\%). Confidence increased from baseline to post two-day course (1.5 to 3.1 on a 4 point Likert scale from Not at all confident (1) to Very confident (4), $p<0.001$ ), and remained unchanged after 1 year (2.73). Course elements correlating with a passing score on the final hands-on test included number of clip uploads ( $r=0.85, p, 0.001)$, attendance at hands-on sessions $(r=0.7, p=0.001)$, and attendance at monthly conferences $(r=0.50, p=0.03)$.

Conclusions: The I-ScaN POCUS training program increased hospitalist knowledge, skill and confidence with maintained skill and confidence after 1 year. Uploading clips and attending hands-on teaching sessions were most correlative with participant success.
\end{abstract}

Keywords: Point-of-care ultrasound, Medical education, Program assessment

\footnotetext{
*Correspondence: janjigian@gmail.com

${ }^{1}$ Department of Medicine, New York University Grossman School of Medicine, NYC Health \& Hospitals/Bellevue, New York, USA

Full list of author information is available at the end of the article
}

(C) The Author(s). 2021 Open Access This article is licensed under a Creative Commons Attribution 4.0 International License, which permits use, sharing, adaptation, distribution and reproduction in any medium or format, as long as you give appropriate credit to the original author(s) and the source, provide a link to the Creative Commons licence, and indicate if changes were made. The images or other third party material in this article are included in the article's Creative Commons licence, unless indicated otherwise in a credit line to the material. If material is not included in the article's Creative Commons licence and your intended use is not permitted by statutory regulation or exceeds the permitted use, you will need to obtain permission directly from the copyright holder. To view a copy of this licence, visit http://creativecommons.org/licenses/by/4.0/ The Creative Commons Public Domain Dedication waiver (http://creativecommons.org/publicdomain/zero/1.0/) applies to the data made available in this article, unless otherwise stated in a credit line to the data. 


\section{Background}

Point-of-care ultrasound (POCUS) is becoming widely adopted across the field of hospital medicine after becoming well established in fields with competency overlap such as Emergency Medicine and Pulmonary and Critical Care Medicine [1, 2]. The reduction in procedural complications, improvement in diagnostic accuracy, and an increase in provider and patient satisfaction has pushed POCUS expansion [3-7]. In addition, the availability of less expensive ultrasound devices and the increasing accessibility of training programs have facilitated the growth of POCUS within general medicine, both in the inpatient and ambulatory settings $[2,8-10]$.

A 2013 survey of internal medicine program directors found that POCUS was considered valuable for diagnostic and procedural use and many residency programs had either adopted formal curricula or had planned to [11]. A similar study of UME leadership in 2014 reported general consensus that POCUS is an important skill worth teaching in medical school [12]. A recent position statement from the Alliance of Academic Internal Medicine recommended the integration of POCUS across the longitudinal training environment for UME, GME and CME in internal medicine [1].

However, the training of faculty has emerged as a key barrier to adoption into hospitalist groups [2, 1315]. Published reports of comprehensive faculty POCUS training programs demonstrate immediate short-term gains as would be expected, however, there is limited data on durability of these gains [1417]. The CHAMP program reported that skills learned in the introductory course tended to wane over time, with participation in monthly scanning sessions/and or portfolio completion being associated with skill retention [14]. The recent position statement issued by the Society of Hospital Medicine (SHM) advocates for attendance at a local or national hands-on training program, which should be followed by a longitudinal study phase with hands-on instruction [2]. Educational outcome data to guide faculty POCUS program development are lacking.

We sought to address concerns of educational durability as we developed and implemented the Integrated Sonography Course at NYU (I-ScaN), a longitudinal POCUS training program for hospitalist faculty. We describe the design, implementation and 1-year outcomes of an observational cohort study of the I-ScaN program.

\section{Methods}

\section{Setting and participants}

The NYU Department of Medicine encompasses four teaching hospitals, NYU Langone Health (Tisch/
Kimmel and Brooklyn campuses), Health + Hospitals/ Bellevue, and the VA New York Harbor Health Care System/Manhattan. Each campus has a unique structure of teaching and non-teaching services and populations served. All physicians who were identified by their directors as core hospitalists, generally defined as those faculty who spend the majority of their clinical time on the inpatient teaching service, were invited to participate. Fifty percent $(23 / 46)$ of the physicians who were asked accepted the invitation to participate.

\section{Program description}

I-ScaN is a faculty development program with the goal of developing a cohort of physicians proficient in POCUS who will foster a culture of ultrasound use across the institution through routine clinical practice of POCUS and who will have the ability to supervise and educate the residents and students in turn. The program consists of four elements: 1 . Introductory course including didactics, image interpretation and hands-on instruction; 2. Supervised practice with patients; 3. Competency assessment; 4 . Skill maintenance and quality assurance [18]. I-ScaN adheres to ultrasound applications and a training structure advocated by SHM [2].

I-ScaN began with a period of self-study upon program registration, where participants were referred to relevant chapters from a POCUS textbook, [18] online videos and selected articles to review. The self-study period lasting approximately 1 month was followed by an intensive two-day course in April of 2018. The course consisted of didactic lectures reviewing theoretical concepts, interactive image-based sessions of interpretation and clinical integration, and hands-on training on human models. To shorten the three-day American College of Chest Physicians Critical Care Ultrasound course upon which the two-day I-ScaN course was modeled,[16] we excluded elements such as the preassessment hands-on testing, critical care application lectures, and the credentialing and economics lecture, while truncating slightly the hands-on sessions. Systems covered in the course included cardiac ultrasound (five standard views), lungs/pleura, abdomen (kidneys, bladder and aorta), and leg vasculature. Participants reviewed both normal and a range of abnormal scans during the Image interpretation sessions.

Following the two-day course, participants began the longitudinal portion of the program. An ultraportable ultrasound device with the ability to upload ultrasound clips to a HIPAA-compliant website was provided to the faculty on each inpatient teaching 
service. Participants also had access to traditional cart-based ultrasound machines at each hospital. Participants were advised to scan patients daily when on service and upload all clips, anonymized to avoid sharing protected health information, to secure servers allowing comments and expert review. For each uploaded clip, participants were asked to provide the anatomic site and a clinical interpretation. The clips were reviewed by an expert (HS) who provided online feedback on both of these domains. Participants used these clips to create a personal portfolio in accordance with national standards set by SHM [19].

Hands-on teaching sessions were led by local experts, primarily Pulmonary and Critical Care Medicine faculty, with a 1: 3 teacher to participant ratio. Sessions typically lasted one hour with the entire time spent at the bedside scanning a patient known to one of the participants. Each of the four hospitals arranged these sessions according to participant and teacher availability with the aim for weekly supervised scanning.

Ultrasound conference, led by the course director (HS), was held monthly with remote viewing capability. Conferences allowed for a participant to present saved clips and a brief lecture to their peer group. Presenters and topics were rotated each month.

\section{Assessments}

We sought to assess participant outcomes at multiple levels of Miller's Pyramid: basic ultrasound knowledge, application of this knowledge to patient care, demonstration of the technical skills of image acquisition, and integration of these new skills into everyday situations [20]. The timeline of program assessments is outlined in the Fig. 1.

Attendance was recorded for monthly conferences, including those who joined remotely, and at hands- on teaching sessions at each site. Total number of uploaded clips was recorded for each participant.

We assessed participants' knowledge of basic ultrasound knowledge, image interpretation, and clinical integration using a novel 20-item online test developed for this program. The same test was administered prior to beginning the program, at the conclusion of the two-day course and again after 1 year. The test was given three times to first measure the impact of the two-day course and then to assess for durability or decay of knowledge over the year.

We evaluated participants' POCUS skills using a hands-on test on human models that was proctored by faculty from the Pulmonary and Critical Care Medicine division. Skills were evaluated by the proctors, using a checklist adapted from the CHEST Certificate of Completion program [21]. The hands-on assessment was rated using a three point scale of poorly done, partially done, or well done. All proctors received an orientation on the assessment tool by the course director. Scores were calculated based on the percentage of items receiving the well done designation.

We assessed participant confidence in image acquisition and clinical integration using a retrospective pre- post- survey at the completion of the two-day course and again after 1 year (Appendix 1). Responses were rated based on a 4-point Likert scale from Not at all confident (1) to Very confident (4).

We also examined participants' barriers to using POCUS. The barrier survey was developed from pilot interviews to address reasons participants may not adopt various aspects of POCUS (i.e. clinical use or clip uploading (Appendix 2)).

Participants completed a questionnaire regarding overall satisfaction with the program and individual elements.

Successful completion of the I-ScaN program required three components: 1) Achieving a score of

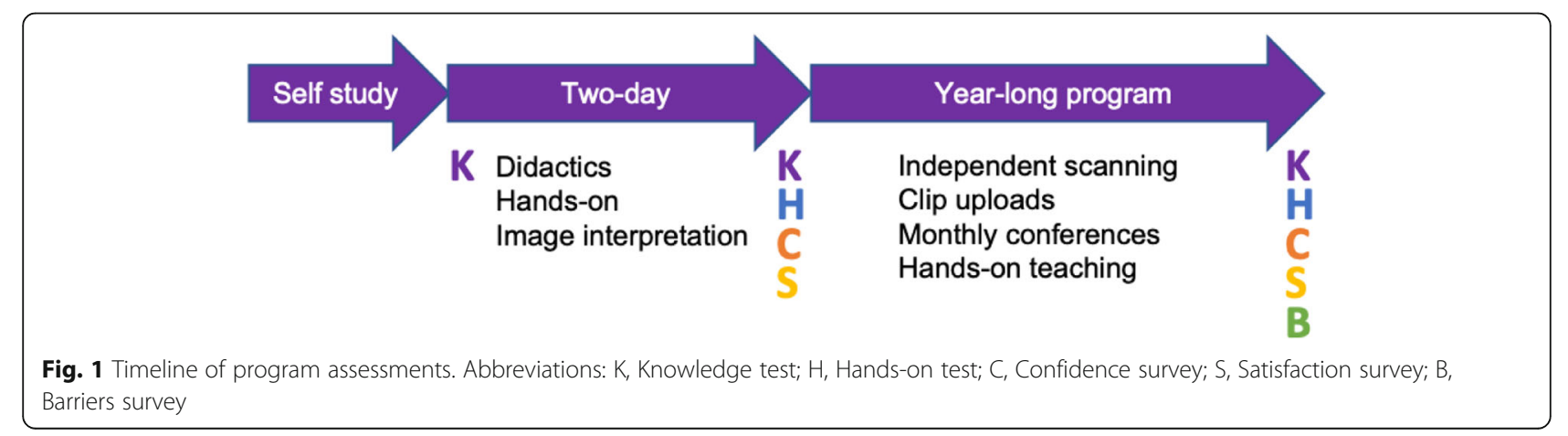


$\geq 80 \%$ on the final knowledge test 2) Receiving a score of $\geq 80 \%$ on the hands-on test and 3) Submitting an image portfolio based on SHM guidance [19]. Passing scores were established at these values through author consensus of clinical relevance and review of published data [14].

\section{Statistical analysis}

Pre- and post- two-day knowledge scores (Cronbach's $\alpha=.68-.74$ ) and confidence in ultrasound use (Cronbach's $\alpha=.79-.84$ ) were summarized. Scores from knowledge were reported as percentages and confidence scores reported on a 1-4-point scale. Differences between pre- and post-values for two-day course variables were assessed by using 2-sample paired Wilcoxon signed rank tests with a 95\% confidence level. Data were reported as median and interquartile range for two-day and year end assessments (Table 1).

Hands-on assessment was conducted at the end of two-days (Cronbach's $\alpha=.84$ ) and at the end of the program (Cronbach's $\alpha=.93$ ). Scores were summarized. At the end of the program, knowledge (Cronbach's $\alpha=.74$ ) and confidence (Cronbach's $\alpha=.91$ ) were reassessed and compared to post-two-days scores. Hands-on and knowledge scores were summarized and reported as percentages, while confidence scores are reported on a 1-4-point scale. Differences between two-days and end of program were assessed by using 2-sample paired Wilcoxon signed rank tests with a 95\% confidence level. Data were reported as median and interquartile range for two-day and year end assessments (Table 1).

Spearman's Correlation was performed on year-end hands-on assessment and knowledge, confidence, lectures, clips uploads, and hand-on lectures to understand the relationship between performance using POCUS and other reported metrics.

We considered a passing score on the final handson test to be the single best summative assessment to determine competency in POCUS skill as participants must demonstrate understanding of the ultrasound machine settings, demonstrate acquisition skills of each view, and identify key structures in each view.

The I-ScaN program qualified as a quality improvement project by the NYU Grossman School of Medicine's Institutional Review Board criteria using a selfcertification process to ensure the data were not collected for research purposes. The primary goal of the project was to assess and improve educational performance of the I-ScaN program.

\section{Results}

Twenty-three hospitalists from across the 4 hospitals participated in the two-day introductory course. Sixteen of the participants (72\%) reported prior ultrasound training, with a range of $2-80 \mathrm{~h}$ (median of $4 \mathrm{~h}$ ); 3 reported more than $5 \mathrm{~h}$ of prior training. Three reported active clinical use of POCUS though none of them had more than $5 \mathrm{~h}$ of prior training. The group averaged 4.5 years of clinical practice (range $=1-13$ years). Only the nineteen hospitalists who completed the assessments at the one-year mark are included in the subsequent analysis (3 left the institution, 1 voluntarily dropped out due to time commitments).

Approximately 1 year after the introductory course and at the time of the final hands-on test, participants had uploaded a total of 2787 clips (range 0-876, median $44)$, one had completed the image portfolio. At the time of writing four participants have satisfied all course elements.

Participant assessment scores from before and after the two-day course are presented in Table 1. Participant knowledge increased from before the two-day course to post-course (58 and $85 \%$ correct, respectively, $p<0.001)$. Knowledge scores fell slightly at 1 year $(81 \%$ correct, $p=0.012)$. There was no change in POCUS skills as measured by the hands-on test from

Table 1 Assessment scores before and after two-day course and after one-year

\begin{tabular}{llll}
\hline & Pre two-day Median (IQR) & Post two-day Median (IQR) & $p$ \\
Knowledge (\% correct) & $58(27)$ & $85(21)$ & $p<0.001$ \\
Confidence (1-4 Likert) & $1.5(0.55)$ & $3.1(0.62)$ & $p<0.001$ \\
& Post two-day Median (IQR) & One-year Median (IQR) & \\
Knowledge (\% correct) & $85(21)$ & $81(24)$ & 0.012 \\
Confidence (1-4 Likert) & $3.1(0.62)$ & $2.73(0.85)$ & NS \\
Hands-on (\% well done) & $75(28)$ & $74(30)$ & NS \\
\hline
\end{tabular}

Abbreviations: IQR interquartile range 
immediately after the two-day course to one-year (75 and $74 \%$, respectively). Confidence ratings increased from 1.5 pre- to 3.1 post-course (out of possible 4, $\mathrm{p}<0.001$ ) and were 2.73 at one-year (NS for incremental change).

Correlation between course components and the hands-on test is presented in Table 2. Performance on the one-year knowledge test correlated highly with results of the one-year hands-on test $(r=0.78$, $p<0.001)$, while correlation between the post-twoday knowledge and hands-on tests was only moderate $(\mathrm{r}=0.53, p=0.018)$. Participant confidence at 1 year positively correlated with hands on performance at 1 year $(\mathrm{r}=0.55, p=0.02)$, however confidence post two-day course did not correlate with hands on performance after the two-day course. There was a correlation between passing the one-year hands-on test with the number of clip uploads $(\mathrm{r}=0.85, p<0.001)$, attending hands-on teaching sessions $(\mathrm{r}=0.7, p=$ 0.001 ), and with attendance at monthly conferences $(\mathrm{r}=0.5, p=0.03)$.

\section{Practice patterns}

At one-year, 13/19 (68\%) of participants strongly agreed or agreed that they felt confident using POCUS to make clinical decisions. Two (11\%) reported using POCUS daily, 7 (37\%) every 2-3 days, 1 (5\%) weekly, and 9 (47\%) "only with the right patient". Lack of time during the workday was reported by $68 \%$ as the principal barrier to clinical use of POCUS. Scanning in front of house staff was reported infrequently as a deterrent ( $21 \%$ agreed or strongly agreed).

In responding to the prompt that "POCUS takes too much time", participants perceived vascular studies to be the most time consuming and lung to be the least (37 and $11 \%$, respectively). Planning to obtain a scheduled formal study was reported as a deterrent to performing POCUS for $21 \%$ of participants for cardiac views and 53\% of participants for vascular views. Only $5-11 \%$ of participants reported not performing POCUS due to the physical exam alone being sufficient to make diagnoses across views.

Participants agreed or strongly agreed that the ultraportable device, when compared to a traditional machine, poses less of a barrier regarding device access (16 and $53 \%$ ), ability to carry/transport (5 and $79 \%$ ), ease of uploading (26 and 74\%), and labeling recorded images (53 and 63\%).

\section{Program evaluation}

At one-year, participants rated I-ScaN with an overall score of 4.6/5. Individual course elements were rated as follows: two-day course 3. 9, hands-on sessions 3. 9, and monthly conferences 3.4 ( 1 not at all useful to 4 very useful).

\section{Discussion}

I-ScaN found that a two-day introductory course was effective at improving hands-on skill, knowledge and confidence, without a decline in hands-on skill following a longitudinal year-long curriculum. The critical portion of successful POCUS skill and knowledge acquisition lies in the longitudinal phase of the curriculum. Participants reported that lack of time was the principal barrier to adoption of POCUS.

Limited experience exists for the ideal POCUS training program for hospitalists. Maw and colleagues described a 10 -week pilot program resulting in immediate improvements in ultrasound acquisition and interpretation though long-term outcomes were not studied [15]. The authors concluded that a longitudinal structured mentored training program would be required for participants to develop mastery. Cochard and others described a hospitalist

Table 2 Correlation between course components and hands-on test

\begin{tabular}{|c|c|c|c|c|}
\hline & \multicolumn{2}{|c|}{ Post two-day hands-on } & \multicolumn{2}{|c|}{ One-year hands-on } \\
\hline & $r$ & $p$ & $r$ & $p$ \\
\hline Post two-day knowledge & 0.53 & $p=0.018$ & & \\
\hline Post-two-day confidence & & NS & & \\
\hline One-year knowledge & & & 0.78 & $p<0.001$ \\
\hline Attendance at hands-on teaching sessions & & & 0.70 & $p=0.001$ \\
\hline Attendance at monthly conference & & & 0.50 & $p=0.03$ \\
\hline Clip uploading & & & 0.85 & $p<0.001$ \\
\hline Confidence at one-year $(n=18)$ & & & 0.55 & $p=0.02$ \\
\hline
\end{tabular}


POCUS training program where, of the 35 completing a survey out of 58 total who took the course, confidence waned at 6 months and 26\% reported never using POCUS in their hospitalist practice [17]. Mathews, reporting on the CHAMP Ultrasound Program, found that of those faculty participating in a voluntary one-day refresher course following the initial three-day introductory course, skill retention was most effectively maintained by those who attended any monthly scanning session or who had started an image portfolio [14]. I-ScaN was designed to maximize the effectiveness of the longitudinal phase while shortening the introductory course to 2 days.

The sample sizes are too small to determine which program elements led to participant success, though similar to the results of the CHAMP program, we found that skill retention on the hands-on test correlated with attendance at monthly conferences, hands-on teaching sessions and image uploading. We believe that incorporation of scanning into clinical workflow immediately after the course along with routine direct supervision are the key elements that result in participant success in POCUS.

Participant confidence in using POCUS at 1 year correlated with their performance on the hands-on test at 1 year but not at the conclusion of the twoday course. These findings suggest that participants developed an awareness of their strengths and weaknesses over time as they progressed along the Dunning-Kruger curve [22]. Provider confidence may serve as a means of assessment when considering an individual's scope of practice who has routinely integrated POCUS into clinical practice.

A strength of our study is the comprehensive data collection for all assessments and surveys of all 19 participants, save a single confidence assessment, representing a diverse faculty across 4 distinct practice settings, including those both highly and minimally engaged during the longitudinal portion of the curriculum. The studies reviewed above either report short-term results or only the highly-engaged fraction of the total cohort of long-term participants.

A recent survey of Internal Medicine faculty addressing barriers to POCUS adoption found that while the majority of respondents held a favorable view of POUCS, the most commonly cited barriers included the need for more training (79\%), lack of a handheld ultrasound device (78\%), lack of direct supervision (65\%), and lack of an expert to review uploaded clips (53\%) [23]. I-ScaN attempted to mitigate common barriers to ultrasound adoption through provision of ultra-portable devices to participants, by bringing teachers to the bedside of participants' patients and providing expert review of all uploaded clips. Responses to our survey did not reveal a clear barrier apart from time, suggesting that I-ScaN was successful in addressing many common barriers. Participants found that the ultra-portable device was less of a barrier than a traditional machine for accessibility, portability, and ability to upload and label clips, supporting resource allocation in favor of these less expensive devices.

Though not specifically addressed on our survey, we believe the low completion rate of the image portfolio was due to the time required to practice, the knowledge gap in how to upload clips, and the lack of an incentive given that successful completion of I-ScaN was voluntary and only required for those who wanted to teach in the program or be allowed by local leadership to use POCUS in clinical care. We have subsequently included uploading of scans as a standard practice during hands-on teaching sessions to address this barrier. We anticipate that uploading of clips and portfolio completion by hospitalists will be commonplace once standards and expectations are set similarly to those found in fields such as Emergency Medicine.

Study limitations include a relatively small sample size encompassing a single cohort of participants, as is common in faculty development programs. The small sample size limits our ability to predict why some individual participants succeeded while others did not. Additionally, while we developed this program to foster a culture of POCUS by training core inpatient teaching faculty, we did not evaluate whether faculty did in-fact teach POCUS to others.

Despite ongoing encouragement and assessment of barriers throughout the longitudinal portion of the course, we found wide variance in faculty participation in program elements and practice patterns. Participants rated all elements of I-ScaN highly, reinforcing that it is the time spent between course elements that predicts durable gains. Getting participants to the bedside following an introductory course to practice on patients and upload clips for feedback will yield the greatest results.

\section{Conclusions}

I-ScaN demonstrates that POCUS skill development learned after a two-day introductory course can be achieved through a longitudinal mentored program consisting of access to ultrasound devices, hands-on teaching sessions, and monthly case conferences. The lowered costs of ultra-portable ultrasound devices and availability of a growing population of instructors will facilitate the expected growth of hospitalists learning POCUS. 


\section{Appendix 1}

Table 3 Retrospective Pre- Post Survey

Prior to taking this program

How confident were you in your ability to: (all responses Not at all confident, Only a little confident, Somewhat confident, Very confident) acquire ultrasound (US) images of the heart?

acquire US images of the lungs?

acquire US images of abdominal organs?

acquire US images of deep veins?

interpret US images of the heart?

interpret US images of the lungs?

interpret US images of abdominal organs?

interpret US images of deep veins?

Prior to taking this program

How likely were you to use ultrasound to? (all responses Not at all likely, Only a little likely, Somewhat likely, Very likely)

evaluate dyspnea?

evaluate abdominal pain?

evaluate hypotension?

evaluate volume status?

evaluate for DVT?

perform a paracentesis?

insert a peripheral venous catheter?

POCUS Questions:

1) Have you received prior training in point of care ultrasound? (Yes/No)

1a) If you answered yes to the question above, how many hours have you received in prior training? (free text)

2) Do you use point of care ultrasound (Yes/No)

Now that you have completed this program

How confident were you in your ability to: (all responses Not at all confident, Only a little confident, Somewhat confident, Very confident) acquire ultrasound (US) images of the heart?

acquire US images of the lungs?

acquire US images of abdominal organs?

acquire US images of deep veins?

interpret US images of the heart?

interpret US images of the lungs?

interpret US images of abdominal organs?

interpret US images of deep veins?

Now that you have completed this program

How likely were you to use ultrasound to? (all responses Not at all likely, Only a little likely, Somewhat likely, Very likely)

evaluate dyspnea?

evaluate abdominal pain?

evaluate hypotension?

evaluate volume status?

evaluate for DVT?

perform a paracentesis?

insert a peripheral venous catheter? 


\section{Appendix 2}

Table 4 I-ScaN One-Year Course Assessment

How useful did you find the following course elements? (all responses Not at all useful, Only a little useful, Somewhat useful, Very useful, N/A) Introductory 2-day course

Monthly conferences

Hands-on teaching sessions

Brightspace site

Do you feel you are competent to use POCUS to make clinical decisions (Strongly Agree, Agree, Disagree, Strongly Disagree)

Overall how would you rate this course? (One of the worst, Below average, Average, Above average, One of the best)

I feel comfortable with my POCUS skills (all responses Strongly Agree, Agree, Disagree, Strongly Disagree)

Abdomen

Cardiac

Lung

Vascular

Performing POCUS takes too much time (all responses Strongly Agree, Agree, Disagree, Strongly Disagree)

\section{Lung \\ Cardiac}

Abdomen

Vascular

I don't use POCUS when I'm going to obtain a formal study (e.g. TTE) (all responses Strongly Agree, Agree, Disagree, Strongly Disagree)

Cardiac

Lung

Abdomen

Vascular

I don't use POCUS because my PE is sufficient to make the diagnosis (all responses Strongly Agree, Agree, Disagree, Strongly Disagree)

Cardiac

Lung

Abdomen

Vascular

A barrier for me with traditional portable US is: (all responses Strongly Agree, Agree, Disagree, Strongly Disagree)

Uploading images

Carrying/transporting US

Labelling images

Access to a device

A barrier for me with Butterfly is: (all responses Strongly Agree, Agree, Disagree, Strongly Disagree)

Uploading images

Carrying/transporting US

Labeling images

Access to a device

I don't feel comfortable using POCUS in front of the house staff (all responses Strongly Agree, Agree, Disagree, Strongly Disagree)

When on service, how often do you scan? (Daily, Every 2-3 days, Once a week, Only with the right patient)

What percentage of scans do you upload? (0-24\%, 25-49\%, 50-74\%, 75-100\%)

What are your barriers to performing US when you are on service? (free text)

What are your barriers to uploading the images? (free text)

How can we help you perform more US and upload more images? (free text)

Did you meet your learning goals? (free text) 
Table 4 I-ScaN One-Year Course Assessment (Continued)

Reflecting on this program, what worked well and what didn't work for you? (free text)

Did you receive sufficient support from this program? (free text)

Did you receive sufficient feedback on your skill development? (free text)

\section{Abbreviations}

POCUS: Point-of-Care Ultrasound; I-ScaN: Integrated Sonography Course at NYU; SHM: Society of Hospital Medicine

\section{Acknowledgements}

The authors would like to acknowledge the Education for Educators (E4E) program, the Program for Medical Education Innovations and Research (PrMEIR), NYSIM, and the Division of General Internal Medicine \& Clinical Innovation.

\section{Authors' contributions}

MJ, AD, CSK, KH, DC, SZ and HS all made substantial contributions to the conception and design of the work, analysis and interpretation of data, and have substantively revised the manuscript draft. All authors have reviewed and approved the manuscript.

\section{Funding}

This program was supported by a generation donation from the Goodman Family Foundation who did not participate in any aspect of this project, including study design, collection, analysis or data interpretation, or in writing of the manuscript.

Declaration of Interest: The authors declare no conflict of interest.

\section{Availability of data and materials}

The datasets during and/or analyzed during the current study available from the corresponding author on reasonable request.

\section{Ethics approval and consent to participate}

The I-ScaN program qualified as a quality improvement project by the NYU Grossman School of Medicine's Institutional Review Board criteria using a self-certification process to ensure the data were not collected for research purposes. The NYU self-certification form that determined this project qualifies as quality improvement has been included in the submission. The primary goal of the project was to assess and improve teaching performance of the I-ScaN program.

Participant consent was not obtained due to the qualification of this project as quality improvement (see attached NYU self-certification form).

\section{Consent for publication}

Not applicable.

\section{Competing interests}

The authors declare that they have no competing interests.

\section{Author details}

${ }^{1}$ Department of Medicine, New York University Grossman School of Medicine, NYC Health \& Hospitals/Bellevue, New York, USA. ²Department of Medicine, New York University Grossman School of Medicine, VA NY Harbo Healthcare System, New York, USA. ${ }^{3}$ Department of Medicine, New York University Grossman School of Medicine, NYU Langone Health, New York, USA.

Received: 13 September 2020 Accepted: 11 December 2020

Published online: 06 January 2021

\section{References}

1. Soni NJ, Lucas BP. Diagnostic point-of-care ultrasound for hospitalists. J Hosp Med. 2014;10(2):120-4. https://doi.org/10.1016/j.amjmed.2019.07.019.

2. Soni NJ, Schnobrich D, Mathews BK, et al. Point-of-Care Ultrasound for Hospitalists: A Position Statement of the Society of Hospital Medicine. J Hosp Med. 2019;14:E1-6. https://doi.org/10.12788/jhm.3079.
3. Soni NJ, Franco R, Velez Ml, et al. Ultrasound in the diagnosis and management of pleural effusions. J Hosp Med. 2015;10(12):811-6. https:// doi.org/10.14423/SMJ.0000000000000833.

4. LoPresti CM, Jensen TP, Dversdal RK, Astiz DJ. Point-of-Care Ultrasound for Internal Medicine Residency Training: A Position Statement from the Alliance of Academic Internal Medicine. Am J Med. 2019;132(11):1356-60. https://doi.org/10.1007/s11606-019-05120-5.

5. Bhagra A, Tierney DM, Sekiguchi H, Soni NJ. Point-of-Care Ultrasonography for Primary Care Physicians and General Internists. Mayo Clin Proc. 2016; 91(12):1811-27. https://doi.org/10.1371/journal.pone.0130066.

6. Mathews BK, Miller PE, Olson APJ. Point-of-Care Ultrasound Improves Shared Diagnostic Understanding Between Patients and Providers. South Med J. 2018;111(7):395-400. https://doi.org/10.1002/jhm.2434.

7. Schnobrich DJ, Gladding S, Olson APJ, Nelson AD. Point-of-Care Ultrasound in Internal Medicine: A National Survey of Educational Leadership. J Grad Med Educ. 2013:5(3):498-502. https://doi.org/10.1111/acem.12435.

8. Bornemann P, Jayasekera N, Bergman K, Ramos M, Gerhart J. Point-of-care ultrasound: Coming soon to primary care? J Fam Pract. 2018;67(2):70-80 http://www.ncbi.nlm.nih.gov/pubmed/29400896. Accessed February 1, 2018.

9. Ye X, Xiao H, Chen B, Zhang S. Accuracy of Lung Ultrasonography versus Chest Radiography for the Diagnosis of Adult Community-Acquired Pneumonia: Review of the Literature and Meta-Analysis. PLOS ONE. 2015; 10(6). doi: https://doi.org/10.1002/jhm.2285.

10. Deeb MA, Barbic S, Featherstone R, Dankoff J, Barbic D. Point-of-care ultrasonography for the diagnosis of acute cardiogenic pulmonary edema in patients presenting with acute dyspnea: a systematic review and metaanalysis. Acad Emerg Med. 2014;21(8):843-52. https://doi.org/10.1016/j. mayocp.2016.08.023

11. Fischer EA, Kinnear B, Sall D, et al. Hospitalist-Operated Compression Ultrasonography: a Point-of-Care Ultrasound Study (HOCUS-POCUS). J Gen Intern Med. 2019:34(10):2062-7. https://doi.org/10.4300/JGME-D-12-00215.1.

12. Mathews BK, Reierson K, Vuong K, et al. The Design and Evaluation of the Comprehensive Hospitalist Assessment and Mentorship with Portfolios (CHAMP) Ultrasound Program. J Hosp Med. 2018;13(8):544-50. https://doi. org/10.1097/ACM.0000000000000414.

13. LoPresti CM, Boyd JS, Schott C, et al. A National Needs Assessment of Pointof-Care Ultrasound Training for Hospitalists. Mayo Clin Proc. 2019;94(9): 1910-2. https://doi.org/10.1016/j.mayocp.2019.07.016

14. Cochard E, Fulkerson Z, Carlos WG. Implementation of a point-of-care ultrasound skills practicum for hospitalists. Ultrasound. 2018;27(1):38-44 https://doi.org/10.12788/jhm.2938.

15. Greenstein YY, Littauer R, Narasimhan M, Mayo PH, Koenig SJ. Effectiveness of a Critical Care Ultrasonography Course. Chest J. 2016;151(1):34-40. https://doi.org/10.3402/meo.v21.33287.

16. Maw A, Jalali C, Khah DJ, et al. Faculty development in point of care ultrasound for internists. Med Educ Online. 2016;21. https://doi.org/10.1016/ j.chest.2016.08.1465.

17. Bahner DP, Goldman E, Way D, Royall NA, Liu YT. The state of ultrasound education in U.S. medical schools: results of a national survey. Acad Med. 2014:89(12):1681-6. https://doi.org/10.1177/1742271X18791317.

18. Soni NJ, Arntfield R, Kory P. Point-of-care ultrasound. 2018. https://www. worldcat.org/title/point-of-care-ultrasound/oclc/1126010674. Accessed 2018.

19. POCUS Certificate of Completion | Society of Hospital Medicine. https:// www.hospitalmedicine.org/clinical-topics/ultrasound/pocus-certificate-ofcompletion/. Published July 6, 2020. Accessed July 6, 2020

20. Miller $\mathrm{G}$. The assessment of clinical skills/competence/performance. Acad Med. 1988;65:63 http://journals.Iww.com/academicmedicine/Abstract/1990/ 09000/The_assessment_of_clinical.45.aspx. Accessed 1988.

21. Critical Care Ultrasonography | Certificate of Completion Program American College of Chest Physicians. https://www.chestnet.org/Education/ 
Advanced-Clinical-Training/Certificate-of-Completion-Program/Critical-CareUltrasonography. Published July 6, 2020. Accessed July 6, 2020.

22. Kruger J, Dunning D. Unskilled and unaware of it: How difficulties in recognizing one's own incompetence lead to inflated self-assessments. J Personal Soc Psychol. 1997;77:1121-34.

23. Wong J, Montague $S$, Wallace $P$, et al. Barriers to learning and using pointof-care ultrasound: a survey of practicing internists in six North American institutions. Ultrasound J. 2020;12(1). https://doi.org/10.1186/S13089-02000167-6.

\section{Publisher's Note}

Springer Nature remains neutral with regard to jurisdictional claims in published maps and institutional affiliations.

Ready to submit your research? Choose BMC and benefit from:

- fast, convenient online submission

- thorough peer review by experienced researchers in your field

- rapid publication on acceptance

- support for research data, including large and complex data types

- gold Open Access which fosters wider collaboration and increased citations

- maximum visibility for your research: over $100 \mathrm{M}$ website views per year

At $\mathrm{BMC}$, research is always in progress.

Learn more biomedcentral.com/submissions 